\title{
Extraperitoneal laparoscopic repair of huge inguinoscrotal bladder hernia: a case report and literature review
}

\author{
HaoHan Chang ${ }^{1}$, Jhen-Hao Jhan ${ }^{2}$, and Sheng Chen Wen ${ }^{3}$ \\ ${ }^{1}$ Affiliation not available \\ ${ }^{2}$ Kaohsiung Medical University \\ ${ }^{3}$ Kaohsiung Medical University Hospital
}

March 1, 2021

\begin{abstract}
Inguinal herniation of urinary bladder is a rare condition which might associated with significant complication. Exact preoperative diagnosis is extremely important. We reported a case of huge inguinoscrotal bladder hernia, associated with bilateral hydronephrosis and kidney injuries, managed by laparoscopy technique.

Extraperitoneal laparoscopic repair of huge inguinoscrotal bladder hernia: a case report and literature review

Abstract

Inguinal herniation of urinary bladder is a rare condition which might associated with significant complication. Exact pre-operative diagnosis is extremely important. We reported a case of huge inguinoscrotal bladder hernia, associated with bilateral hydronephrosis and kidney injuries, managed by laparoscopy technique.
\end{abstract}

Keywords : Bladder, Hernia, laparoscopic, repair, scrotal cystocele

\section{Introduction}

Bladder, despite approaching to inguinal region, rarely drops into inguinal canal causing bladder herniation. Bladder herniation only accounted for 1-3\% of all inguinal herniation ${ }^{1}$, while the incidence may reach up to $10 \%$ among obese patient over 50 years old ${ }^{2}$. Most of the cases are generally asymptomatic, while the advanced cases must complete two-stage urination by compressing scrotal-bladder manually ${ }^{3}$. As most of the hernia are diagnosed by physical examination without advance radiology exam prior to operation, only $7 \%$ of inguinal bladder hernia is diagnosed before the herniorraphy. Therefore, $16 \%$ of these condition are found by intraoperative or postoperative complication such as bladder injury and bladder leakage ${ }^{4}$. In this manuscript, we reported a bladder herniation diagnosed prior to operation and repaired by laparoscopic extraperitoneal approach (TEP) herniarraphy.

\section{Case report}

A 82-year-old man with chronic urinary frequency and incomplete voiding presented fever and chillness for 3 days. Physical examination revealed a large, right side scrotal mass which existed for more than ten years, according to the patient's statement. The size of mass reduced to some degree after voiding. Kidney echo revealed bilateral hydronephrosis and hydroureter. Urinary tract infection and acute kidney injuries were diagnosed by blood exam and urine analysis. Foley catheter was placed in order to relieve his voiding symptoms. Computed tomography revealed right inguinoscrotal hernia containing a great portion of bladder 
(Panel A, arrow), while bilateral ureterovesical junction were stuck inside the scrotum, resulting in bilateral hydronephrosis and hydroureter. After a course of levofloxacin treatment, we arranged total extraperitoneal laparoscopic inguinal hernia repair. During operation, right direct type inguinoscrotal bladder hernia was discovered (Panel B, arrow), while left side direct type inguinal hernia was also noted. After gently pulled the hernia sac and the bladder, we placed an anatomical mesh at each side to fill up the defect. The patient discharge on post-operative day 1 , while his urinary complaint reduced significantly after operation. During further follow up, there was no more hydronephrosis noted through kidney, his renal function also improved as well.

\section{Discussion}

Bladder hernia is a rare condition, accounting for 1-3\% of inguinal hernia ${ }^{5}$. Most of bladder heriation occur on the right side and are direct type. ${ }^{1}$ The pathophysiology of an inguinal bladder herniation was similar with direct type inguinal hernia, as the sheath of peritoneum traverse through the weak point of abdominal fascia ${ }^{5}$. Some risk factors are associated with this condition, including obesity, weakness of abdominal musculature, chronic urinary obstruction, male gender, old age and benign prostatic hypertrophy ${ }^{5,6}$. Most of the patient are typically asymptomatic while some of them may be suffered by lower urinary tract symptoms (LUTS). In advance cases, patient may describe two-stage voiding as they have to complete urination by compressing scrotal-bladder manually ${ }^{7}$. As occurred in our case, previous reports have shown that some patient might developed bilateral hydronephrosis, acute kidney injury and ureteral obstruction resulting from bladder compression $^{7,8}$.

There are several examinations could be used for diagnosing bladder herniation. Besides physically examination, sonography provided another non-invasive, non-radiation method which may demonstrate a hypoechoic mass inside the scrotum. The size of the mass may decrease after voiding or Foley indwelling. Sonography could also be used for screening of hydronephrosis and hydroureter. Voiding cystourethrography may revealed a dog-ear shape of bladder inside the scrotum, providing adequate evidence for diagnosis. ${ }^{1}$ Computed tomography (CT) scan could provide most detailed information for diagnostic purpose and surgical planning, including whether there is contralateral herniation or other organ herniation simultaneously.

While radiology imaging is not routinely performed in the workup of inguinal hernia, only $7 \%$ of inguinal bladder hernias are diagnosed before operation. Furthermore, complication such as bladder injury or bladder leakage may occurred during surgery, accounting for $16-23.5 \%$ of all bladder hernia. ${ }^{4,9}$ Thus, it is important to identify bladder hernia prior to operation.

The most frequently reported surgical way of treating bladder hernia is open repair, yet our patient underwent a laparoscopic total extraperitoneal approach (TEP) hernia repair. Laparoscopic surgery provides several advantages including improving visibility of bladder and surrounding structures, rapid recover, less pain and improved cosmesis. ${ }^{10}$ Currently, there were several published literatures reporting laparoscopic repair of herniated bladder, however, in best of our knowledge, non-of them provide intra-operative image clearly.

\section{Conclusion}

Herniation of bladder is a rare clinical condition, consisting of potentially prominent complication, hiding in a very common surgical indication-inguinal herniation. Detailed history and examination for exact diagnosis are extremely important. While most of the previous reports suggested open approach as the way of surgical repair, we confirmed that these situations might be managed safely by laparoscopic total extraperitoneal approach. Laparoscopic approach offers similar surgical outcome as well as better visibility and improved cosmesis.

Figure 1. Bladder and bilateral ureter herniation associated with bilateral hydronephrosis

Figure 2. Intra-operative image, showing bladder herniation and surrounding structures

1 Hamidi Madani A, Mohammadi Nikouei H, Baghani Aval H, Enshaei A, Asadollahzade A, Esmaeili S. Scrotal herniation of bladder: a case report. Iran J Med Sci 2013; 38 :62-64. 
$2 \mathrm{Kim} \mathrm{KH}, \mathrm{Kim} \mathrm{MU}$, Jeong WJ et al. Incidentally detected inguinoscrotal bladder hernia. Korean J Urol $2011 ; 52: 71-73$.

3 Kraft KH, Sweeney S, Fink AS, Ritenour CW, Issa MM. Inguinoscrotal bladder hernias: report of a series and review of the literature.Can Urol Assoc $J$ 2008; 2 :619-623.

4 Khan K, Chaudhry A, Feinman MB. Inguinoscrotal hernia containing the urinary bladder. BMJ Case Rep 2016; 2016 .

5 Elkbuli A, Narvel RI, McKenney M, Boneva D. Inguinal bladder hernia: A case report and literature review. Int J Surg Case Rep 2019;58:208-211.

6 Moufid K, Touiti D, Mohamed L. Inguinal bladder hernia: four case analyses. Rev Urol 2013; 15 :32-36.

7 Wagner AA, Arcand P, Bamberger MH. Acute renal failure resulting from huge inguinal bladder hernia. Urology 2004; 64 :156-157.

8 Habib A. A Rare Case of Inguinal Hernia with Complete Bladder Herniation. Case Rep Surg 2017; 2017 :4658169.

9 Oruc MT, Akbulut Z, Ozozan O, Coskun F. Urological findings in inguinal hernias: a case report and review of the literature.Hernia 2004; 8 :76-79.

10 Khan A, Beckley I, Dobbins B, Rogawski KM. Laparoscopic repair of massive inguinal hernia containing the urinary bladder. Urol Ann2014; 6 :159-162.

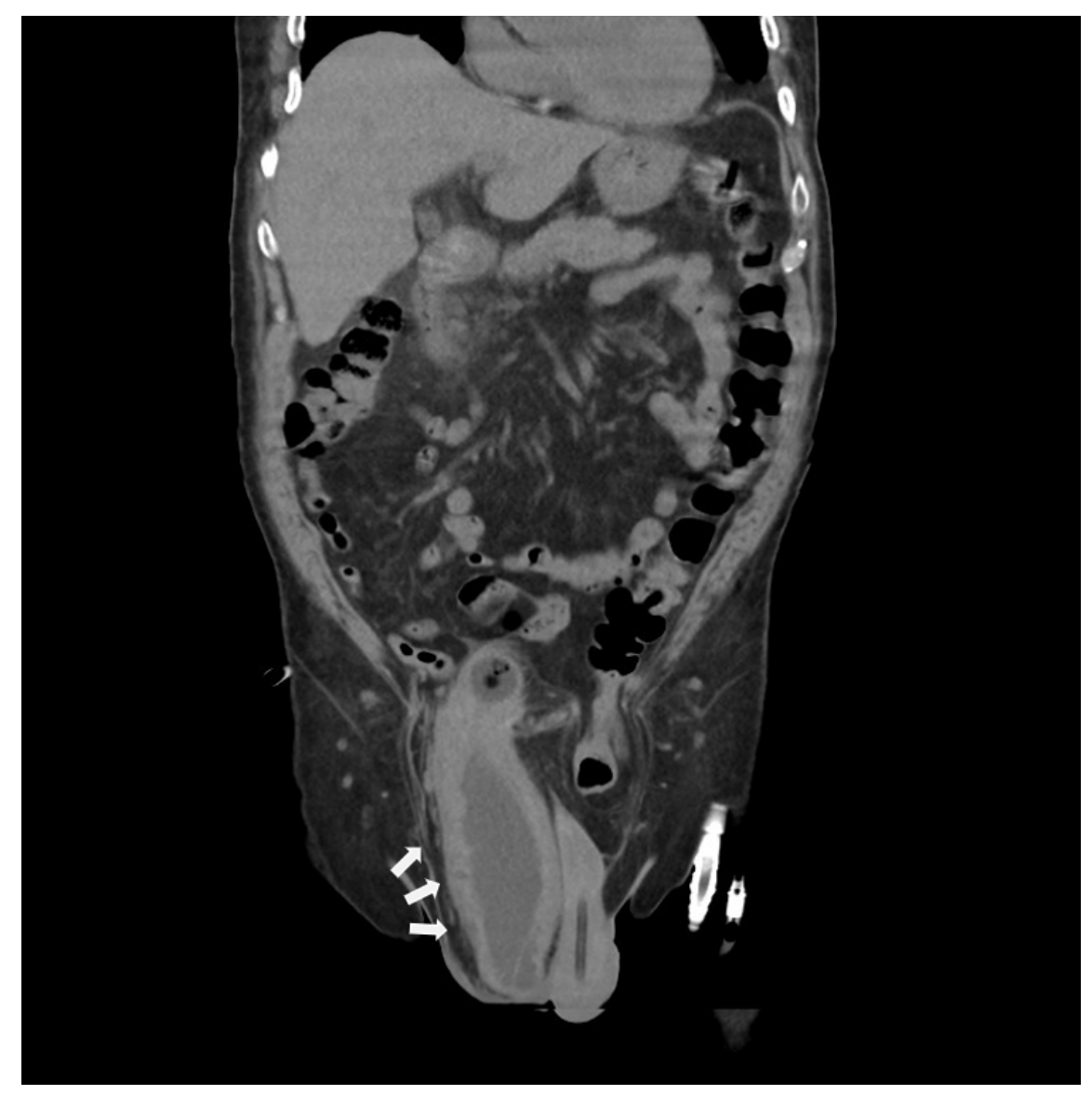




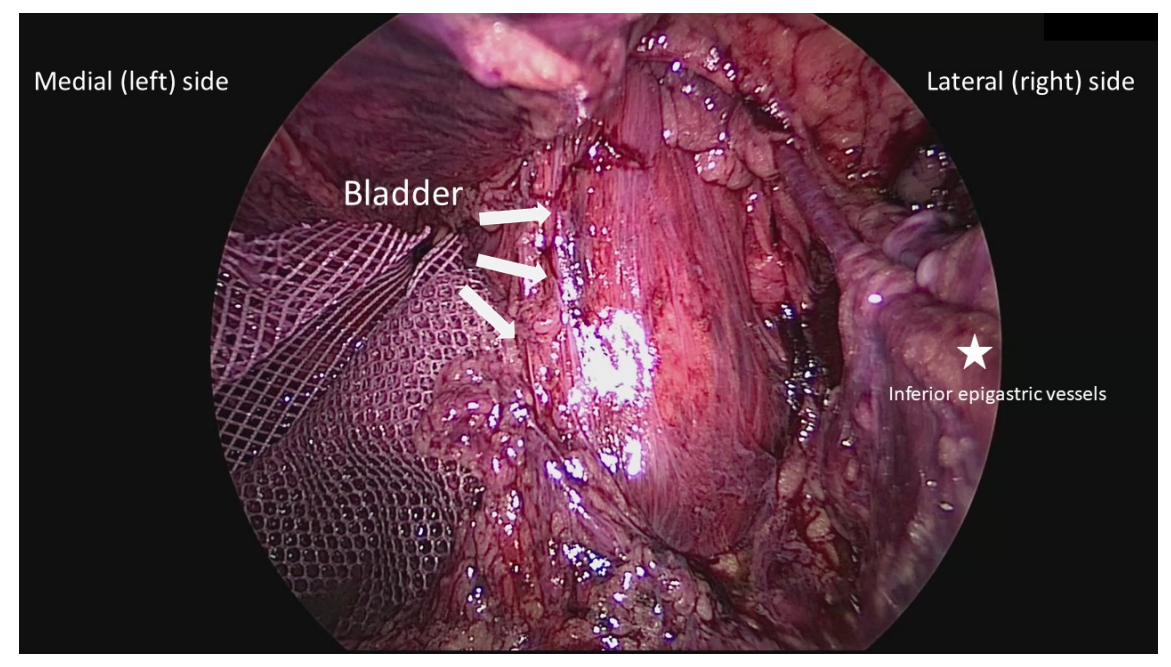

